\title{
Lithography of Curvature
}

\author{
Eran Sharon, The Racah Institute of Physics
}

The Hebrew University of Jerusalem, Jerusalem,

Israel

Most manmade machines change configurations via rotation or translation of rigid elements, or a "skeleton". In some cases flexible elements, such as membranes, are attached to the skeleton and are passively deformed by its motion. Such rigid machines are common in nature as well, as practically the same principles govern the motion of Vertebrates.

Nature is full with another kind of shape transforming structures that can be called "soft machines": An octopus, worms or even growing plants, change their shapes in time via active deformation of their tissue. In such shaping method, each element of the tissue actively "aims" to a new, stress-free, reference "state", with a given infinitesimal volume and orientation. The outcome of all these active local deformation is a new global configuration of the entire body. Despite its great applicative potential, such "shaping by active deformation" has not yet been implemented into a design technique, mainly due to theoretical and experimental difficulties. In recent years there has been a progress in both theoretical formulation and the development of experimental and implementation techniques. A recent work by Kim, Hanna, Byun, Santangelo and Hayward (Science 335, 1201, 2012) marks an important step in turning "shaping by active deformation" into design and production technique.

An elastic body that undergoes non-uniform growth or swelling is likely to contain residual stresses - Its elements in their new reference state (or shape) would not fit together. Since the elements are "glued" together the entire body assumes a new configuration. This configuration minimizes the elastic energy, but does not zero it (As the "elements" do not fit, they are locally deformed with respect to their new reference state, even when the body is free of external load). What would be the selected global configuration of the body is not an easy question to answer. Alternatively, what is the swelling distribution that will lead to a specific global configuration is another difficult problem. Finally, even if we know the answers we are left with the big problem of how to construct soft material that undergoes the desired, non-uniform swelling.

During the last decade there has been an intensive study of actively deforming thin elastic sheets. In particular, objects named Non-Euclidean Plates (NEP) were defined and studied [1]. NEPs are thin plates that undergo some lateral growth or swelling that is uniform across the thickness of the plate, but varies with location within the plate. The term Non-Euclidean expresses the fact that in these plates the reference lengths 
following the swelling cannot be realized as a flat surface. They are described by a non-Euclidean "reference metric tensor", , which by Gauss "Theorema egregium" determines a non zero Gaussian curvature. This implies that any configuration of the sheet, which is free of in-plane strains, cannot be flat. Given some reference metric, an elastic sheet, which is free of external constraints will buckle into a 3D configuration, in which the reference lengths are approximately fulfilled. Such buckling costs bending energy and therefore, the selection of a configuration is based on the competition between stretching and bending. The theory of NEP uses the framework of differential geometry to express the reference lengths and the energy of such sheets, allowing the calculation of equilibrium configurations of NEPs.

In order to build responsive Non-Euclidean Plates, one has to construct a thin sheet made of a flexible material that can undergo differential swelling/shrinkage upon induction with external stimuli. Responsive gels are excellent candidates for such tasks. First samples of responsive NEP were made of $\mathrm{N}$ - isopropylacrylamide (NIPA) - a hydro gel that undergoes volume reduction transition at temperature higher than $33 \mathrm{C}^{0}$ - with non-uniform monomer concentration. However, this technique was limited in the range of metrics that can be produced, and could not be developed into a manufacturing technique. Kim et.al. have made a big step forward by developing a technique that can be called 'Lythography of curvature". They used selective UV crosslinking of NIPA gel as a way for determining local swelling ratios: Swelling/ shrinkage of a highly crosslinked gel is minor, while a weakly crosslinked gel undergoes large volume changes when the temperature is varied. In the experiments partially crosslinked NIPA polymer sheets were further crosslinked by exposing them to UV light through a mask that consists of micron size opaque dots with varying diameter. This process "prints" on the sheets spots that shrink a lot (opaque dots), or little (clear mask). The actual reference metric is very bumpy; containing sharp gradients arranged in an array of "blisters". However, as long as the sheet is not too thin, elasticity itself "coarse grains" into an effective, smooth reference metric. Regions containing large dots tend to shrink more than those with small dots and in a way a "gray level" determines the local swelling/shrinkage ratios.

With this technique the authors generated NEPs that assumed several pre-programmed geometrical shapes, such as cones, surfaces with constant Gaussian curvature and minimal surfaces. The nontrivial work of calculation of swelling profiles, production of NEPs and measurements of their shapes was performed on gel discs of few hundred microns in diameter. Even in such a small scale the authors showed impressive quantitative agreement between experiments and theoretical predictions. In this sense, shaping via active deformation can be regarded as an existing design technique in the sub-millimeter scale.

Shaping of NEPs has some unique characteristics. A 2D printed "picture" is converted into a 3D shape via a unique connection. The picture does not prescribe a shape. It only prescribes a reference metric. Since most metrics have many different realizations 
as surfaces, NEPS have an unusual flexibility in selecting highly different configurations. Minor changes in can lead to dramatic global shape changes. This property is beneficial when designing shape transforming surfaces. However, this flexibility makes the control over shape selection very difficult: For a given some very different shapes are nearly energetically degenerate. One can activate an NEP to get a hat and instead get a cat... It is likely that "assisting" NEP via small variation in the bending term could increase reliability, while keeping some of the flexibility.

The connection between the "picture" and the "shape" of a sheet is often surprising As the only prescribed entity is, the range of accessible configurations by very thin sheets is tightly related to the group of isometric embedding of in Euclidean space. It turns out that some very simple reference metrics would lead to complicated 3D shapes.

The physics of NEPS has a broad relevance. In principle, NEPs can be constructed from other materials. Nematic elastomers and electro active polymers are such potential materials. In addition, the underlying geometrical principles that govern shaping via active deformation are possibly relevant to the shaping of self-assembled chemical supra structures. The local angles and lengths, determined by chemical "bonds" prescribe a reference metric. It is an interesting question, which structures are "soft" capable of compromising with respect to - and which are "brittle". Finally, the physics of shaping via active deformation allows for a better understanding of naturally evolving systems, such as invertebrate's motion and morphogenesis during growth. A highly recommended paper, which studies alteration of leaf shapes is "Genetic control of surface curvature" (Nath et.al , Science 299, 1404, 2003). 


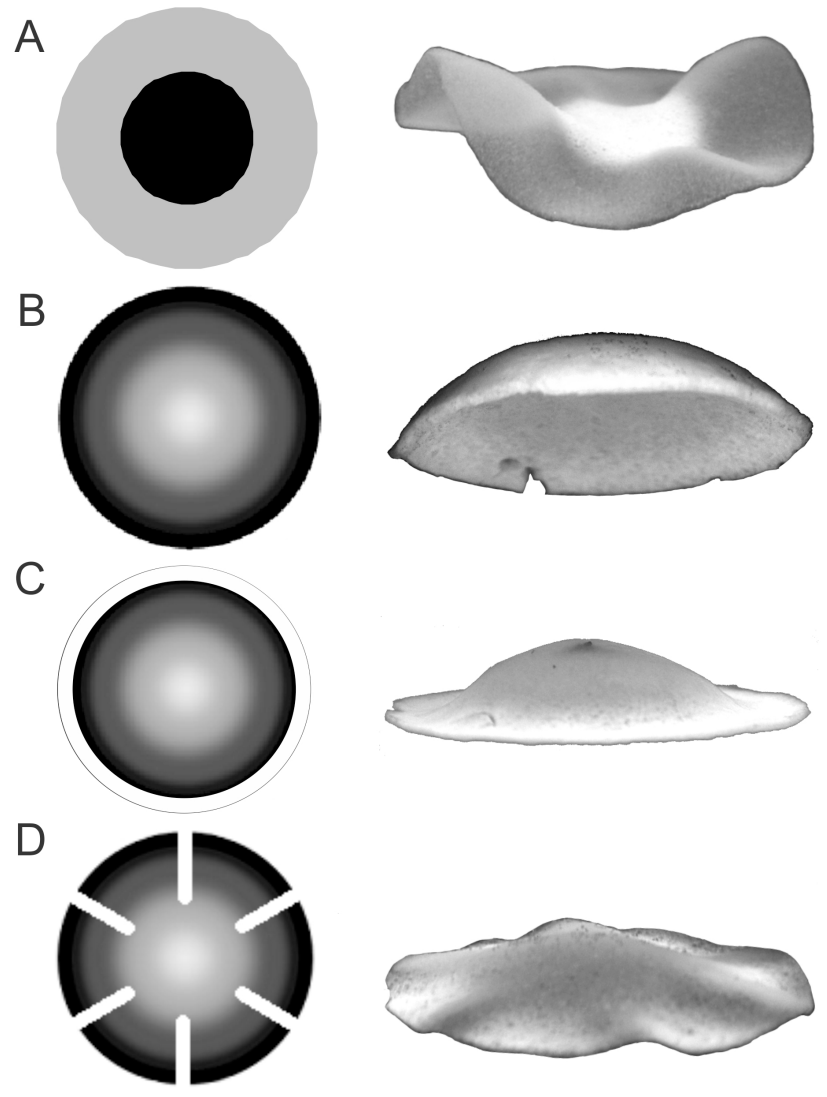

Figure1: The connection between 2D masks and 3D configurations. Examples of masks (left) and the resultant buckled NIPA gel sheets (right). In these macro scale samples the local "gray level" of the mask determines the amount of UV irradiation onto different regions of the sheet. This determines the crosslinking density. Once the plates are activated in a hot bath the crosslinking "map" is converted into non-uniform shrinkage, which leads to the buildup of a 3D shape. (A) A dark center region leads to an enhanced shrinkage in the center and the formation of a wavy disc. (B) A continuous gradient of gray scale can be tuned to construct a spherical dome shape. Small changes of the mask in (B) in the form of a clear ring along the margins (C), or six radial clear lines (D) leads to different modulations of the spherical dome. Mask diameter is $5 \mathrm{~cm}$. Samples were made by Ido Levine.

[1] E. Efrati, E. Sharon, and R. Kupferman, Jour. Mech. Phys. Solids 57, 762 (2009).

[2] T. Tanaka, Physical Review Letters 40, 820 (1978).

[3] U. Nath et al., Science 299, 1404 (2003). 